\title{
Performance of Implementing Smart DFT in Micro Phasor Measurement Unit
}

\author{
Lai Chao-Yuan ${ }^{1}$, Yang Tien-Yen ${ }^{1}$, and Liu Chih-Wen ${ }^{1}$ \\ ${ }^{1}$ Department of Electrical Engineering, National Taiwan University, Taipei, Taiwan
}

\begin{abstract}
The advance of the signal processing, this paper implement a more accurate digital measurement algorithm, Smart DFT(SDFT) in Micro Phasor Measurement Unit( $\mu$ PMU), which is based on Discrete Fourier Transforms(DFT) to estimate frequency information from Taiwan power system. $\mu$ PMU, the sensor we plan to acquire the information from the power system by using the signal of outlet voltage-level $110 \mathrm{~V}$ in Taiwan, such as frequency, voltage and angle. The performance of SDFT implemented in $\mu$ PMU represents a more precise frequency information when frequency fluctuation occurred just as the frequency of power system. We offer the results of simulations, stable frequency generated from waveform generator and real frequency from main electricity to compare SDFT with DFT method which implemented in $\mu$ PMU.
\end{abstract}

\section{Introduction}

Transition to Smart Grid, time synchronized sensor of the power system is indispensable. With time synchronized sensor the system can analysis data in near real time without time correction for different sensor or devices, which takes plenty of time in the past. The growing penetration of renewable energy, complexity of the power grid and electricity demand, the reliability of the power system will be more difficult and important issue to face with. Moreover, most of the industries in Taiwan are high-tech company, they cannot afford the huge lost which cause by the unexpected power event from the grid. According to the needed of maintaining the reliability of the power system in a more complex power system, Wide Area Measurement System(WAMS) has been proposed [1], [2]. The power grid WAMS has been elongated from the transmission level to the distribution level all over the area, and the rate of uploading frequency information has been upgrade greatly. This could lead the system react and move forward to control the system faster than conventional supervisory control and data acquisition(SCADA) system. To build WAMS the sensors are needed, Micro Phasor Measurement Unit( $\mu$ PMU), a measurement unit which is comparable with Phasor Measurement Unit, but single phase and in a low voltage level which is chosen to implement in Taiwan Wide Area Measurement System(T-WAMS). Due to the more accurate frequency WAMS adopted the more precise decisions the system or operators could make, Smart Discrete Fourier Transforms(SDFT) [3], [4]. SDFT the digital measurement algorithm we chose to implement in $\mu \mathrm{PMU}$ obtains a more precise frequency value than conventional Discrete Fourier
Transforms(DFT) when frequency deviates from the standard frequency. Moreover, SDFT is the extend of DFT, the benefits of DFT are kept, such as, completely filter the integer harmonic and the recursive calculation. The remainder of the paper is organized in the following manner. Section II introduces the architecture of selfdevelopment $\mu \mathrm{PMU}$ and the digital algorithm, SDFT, we implemented in $\mu$ PMU. Section III discusses the performance of SDFT in non-standard frequency that generate by waveform generator. Section IV provides conclusions and directions for further research.

\section{Architecture \& algorithm implemented in $\mu \mathrm{PMU}$}

\subsection{Architecture}

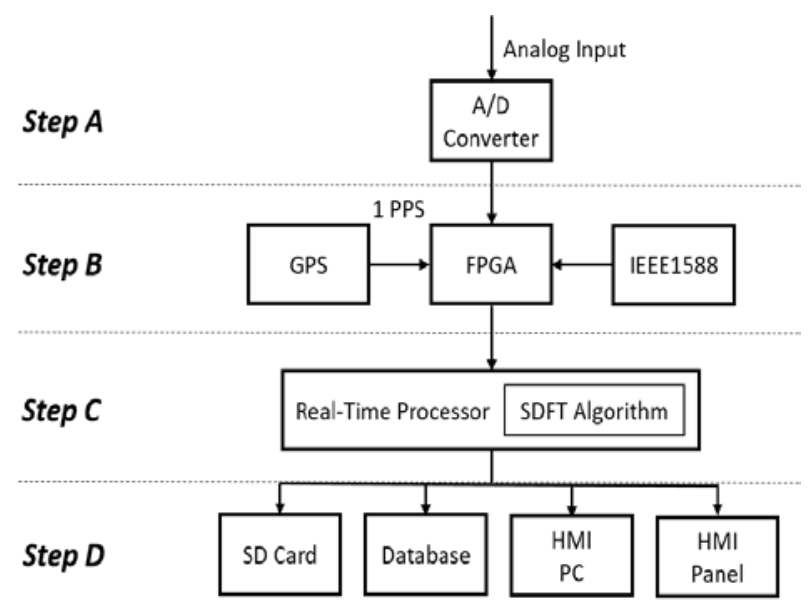

Fig. 1. Caption of the Figure 1. Below the figure.

\footnotetext{
* Corresponding author: D05921011@ntu.edu.tw
} 
Process procedure of self-develop $\mu \mathrm{PMU}$ shown in Figure1 are separated in 4 part, Step A to Step D. Step A represents the analog input signal which came from the outlet, 110AC. The synchronize mechanism in Step B is one of the most important technique, due to the technique

of time synchronization, data from different locations of $\mu$ PMU could be well analysis in real-time. The synchronization mechanism in the $\mu \mathrm{PMU}$ we built is a dual-time $\mu \mathrm{PMU}$ with applying Global Positioning System(GPS) and IEEE1588v2 time synchronization resources [5]. After combining timestamp with the raw data obtained from the $110 \mathrm{~V}$ outlet, the calculation of frequency comes up in Step C, which SDFT algorithm implements. The last part of the procedure is Step D, which transmits data, such as the information of power grid to phasor data concentrator(PDC). Furthermore, convenient Human Machine Interface(HMI) touch panel are also considered.

\subsection{Algorithm}

\section{1) DFT}

Discrete Fourier Transforms is used for analyzing discrete-time finite-duration signals in the frequency domain. With the feature of recursive, one of the advantage of DFT is that it can calculate efficiently. The other advantage of DFT is that it can immune the harmonics of standard frequency, but as the frequency deviates the calculation of frequency becomes inaccurate.

\section{2) SDFT}

Smart Discrete Fourier Transforms, a digital measurement algorithm which is based on DFT but get over the leakage error cause by DFT when the frequency offset occurs. SDFT keeps all the advantage of DFT such as fast calculation and eliminate the harmonics. Moreover, overcomes the leakage error. This breakthrough is important to the power system, because when energy unbalance event occurs, the frequency may differ rapidly. The incorrect frequency information may cause the system or the operators make a wrong decision, and lead the power system into a dangerous situation.

\section{Testing Data}

To discover the difference between DFT and SDFT, we implement SDFT and DFT algorithm into two selfdeveloped $\mu$ PMU. The consisting A/D converter hardware in $\mu$ PMU is a $50 \mathrm{kS} / \mathrm{s}$ sampling rate with having 250 Vrms measurement range data acquisition module. To compare DFT and SDFT, the testing environment consists two $\mu$ PMUs. One of the $\mu \mathrm{PMU}$ implemented with SDFT, the other implemented with NI DFT function (DFT function created by National Instrument). The input waveform is generated by the 33500B series waveform generator frequency resource from the frequency range $57 \mathrm{~Hz}$ to $63 \mathrm{~Hz}$. $\mathrm{x}(\mathrm{t})=$ $5 \cos (2 \pi f t), f=57 \mathrm{~Hz}$ to $63 \mathrm{~Hz})$

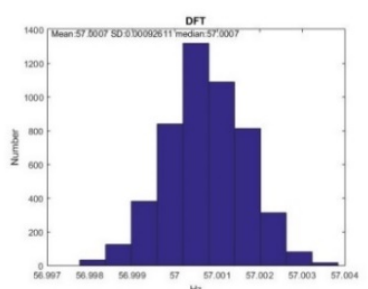

(a)

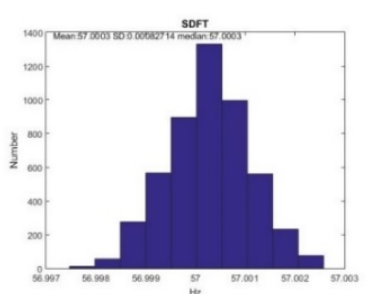

(b)
Figure 2. (a) DFT and (b) SDFT with $x(t)=5 \cos (2 \pi 57 t)$

Table 1. Mean, median and standard deviation with $\mathrm{x}(\mathrm{t})=5 \cos (2 \pi 57 t)$

\begin{tabular}{|c|c|c|}
\hline $57 \mathrm{~Hz}$ & MEAN & \\
\hline DFT & 57.0007 & \\
\hline SDFT & 57.0003 & \\
\hline
\end{tabular}

(a)

\begin{tabular}{|c|c|} 
MEDIAN & $\begin{array}{c}\text { STANDARD } \\
\text { DEVIATION }\end{array}$ \\
\hline 57.0007 & 0.000926 \\
\hline 57.0003 & 0.00082714 \\
\hline
\end{tabular}

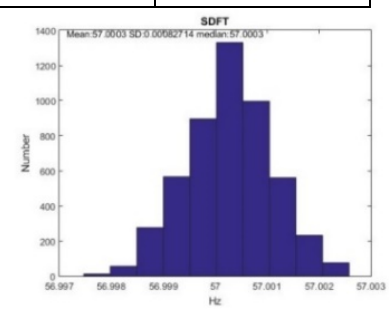

(b)
Figure 3. (a) DFT and (b) SDFT with $\mathrm{x}(\mathrm{t})=5 \cos (2 \pi 60 t)$

Table 2. Mean, median and standard deviation with $\mathrm{x}(\mathrm{t})=5 \cos (2 \pi 60 t)$

\begin{tabular}{|c|c|c|c|}
\hline $60 \mathrm{~Hz}$ & MEAN & MEDIAN & $\begin{array}{c}\text { STANDARD } \\
\text { DEVIATION }\end{array}$ \\
\hline DFT & 60.0009 & 60.0009 & 0.00099625 \\
\hline SDFT & 60.0004 & 60.0004 & 0.00066653 \\
\hline
\end{tabular}

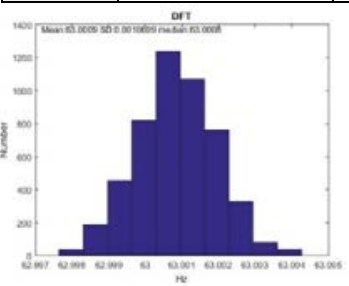

(a)

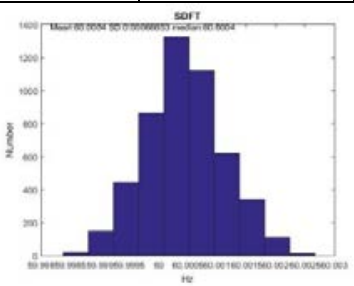

(b)
Figure 4. (a) DFT and (b) SDFT with $\mathrm{x}(\mathrm{t})=5 \cos (2 \pi 63 t)$

Table 3. Mean, median and standard deviation with $x(t)=5 \cos (2 \pi 63 t)$

\begin{tabular}{|c|c|c|c|}
\hline $63 \mathrm{~Hz}$ & MEAN & MEDIAN & $\begin{array}{c}\text { STANDARD } \\
\text { DEVIATION }\end{array}$ \\
\hline DFT & 63.0008 & 63.0008 & 0.0010699 \\
\hline SDFT & 63.0006 & 63.0006 & 0.0007028 \\
\hline
\end{tabular}

The vertical axis of Figure 2 to Figure 4 is the counting number of the frequency which falls in the frequency range, and the horizontal axis represents the 
frequency range which $\mu$ PMU calculated. From the testing result of the figure, we can tell that the figure generated from SDFT is narrower than the figure generated from DFT, which is stand for the frequency deviation calculated by SDFT is less than DFT. From the information in table I to table III, such as, mean, median and standard deviation, could clearly tell that the frequency calculated by SDFT is much more precise than the frequency calculated by NI DFT.

On the other hand, we also tested the performance of DFT and SDFT on MATLAB, when the sampling rate are setting at $2400 \mathrm{~Hz}$ and $2000 \mathrm{~Hz}$. The standard frequency in Taiwan is operating at $60 \mathrm{~Hz}$, as the DFT algorithm, the sampling rate of DFT must be the multiple of $60 \mathrm{~Hz}$. In the case which sampling rate is set to $2400 \mathrm{~Hz}$, the frequency result of DFT have a larger oscillation than the result calculated by SDFT, but even DFT oscillates larger than SDFT, it still presents a small deviate from standard frequency, $60 \mathrm{~Hz}$. Without sampling in the multiple of $60 \mathrm{~Hz}$, DFT presents a huge oscillation, and the frequency calculated by SDFT stays in an outstanding performance $60 \mathrm{~Hz}$, which is shown in figure 6 .

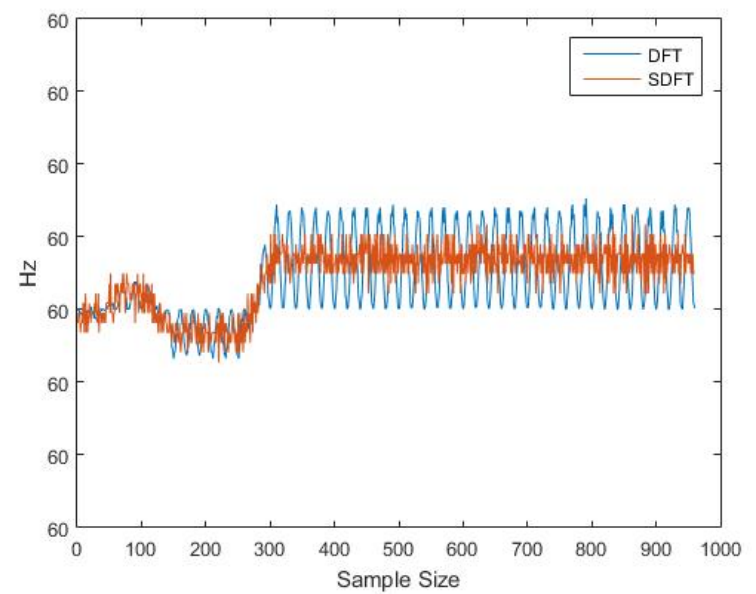

Figure 5. Compare DFT and SDFT with $2400 \mathrm{~Hz}$ sampling rate

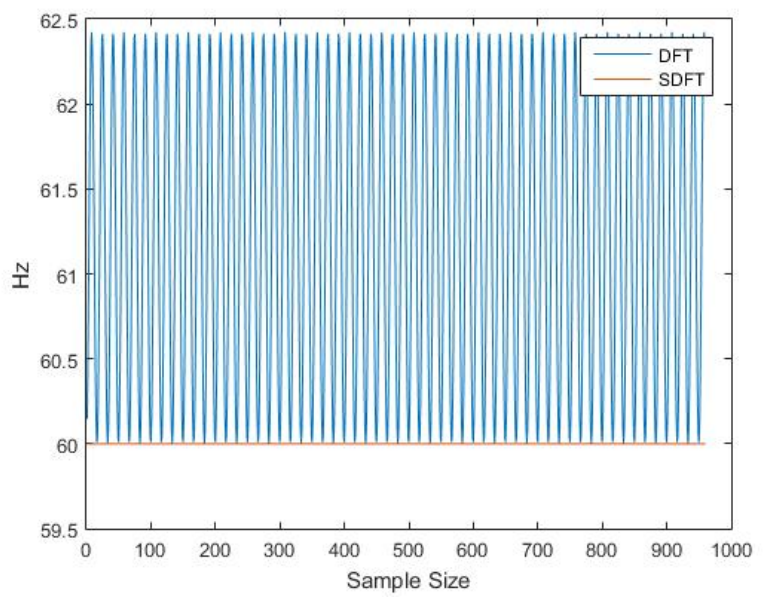

Figure 6. Compare DFT and SDFT with $2000 \mathrm{~Hz}$ sampling rate

The testing result shows in figure 6 presents that SDFT is able to calculate accurate frequency without using a multiple of $60 \mathrm{~Hz}$ sampling rate. This overcome is important to the hardware which do not have the exactly sampling rate which the implement algorithm needed.

\section{Conclusions \& future work}

An intelligent digital algorithm, SDFT, has been implement in a self-development micro phasor measurement unit and verify in this study. By implementing SDFT, the performance of the frequency calculation gives an outstanding performance than the conventional DFT algorithm. Such as 1) the result which calculated frequency from SDFT when the frequency deviates from the standard frequency, and 2) the calculation result when the sampling rate is not the multiple of $60 \mathrm{~Hz}$. Last but not least, SDFT keeps the advantages of DFT, such as calculate efficiently with the recursive equation and could immune the harmonics of standard frequency. The calculation of the precise frequency is an important issue in power system, especially when supply and demand side are not balance which cause the frequency of power system deviates.

\section{References}

1. M. Akke and D. Karlsson, "Phasor measurement applications in Scandinavia," in Transmission and Distribution Conference and Exhibition 2002: Asia Pacific. IEEE/PES, pp. 480-484, 2002.

2. J. W. Ballance, B. Bhargava, and G. D. Rodriguez, "Monitoring power system dynamics using phasor measurement technology for power system dynamic security assessment," in Power Tech Conference Proceedings, 2003 IEEE Bologna, 2003, p.7 pp. Vol. 3.

3. Jun-Zhe Yang and Chih-Wen Liu,'A precise calculation of power system frequency and phasor," IEEE Trans. Power Del., vol. 16, no. 3, pp. 361-366, Jul.2001

4. J.-Z. Yang, C.-W. Liu, “A precise calculation of power system frequency and phasor," IEEE Trans. Power Del., vol. 15, no. 2, pp. 494- 499, Apr 2000.

5. Ching-Chuan Luo and Chih-Wen Liu,” Design and implementation of dual time synchronization signal for Micro Phasor Measurement Unit ( $\mu$ PMU)," in Instrumentation and Measurement Tech Conference Proceedings, 2016 IEEE International, pp. 1-6, 2016 